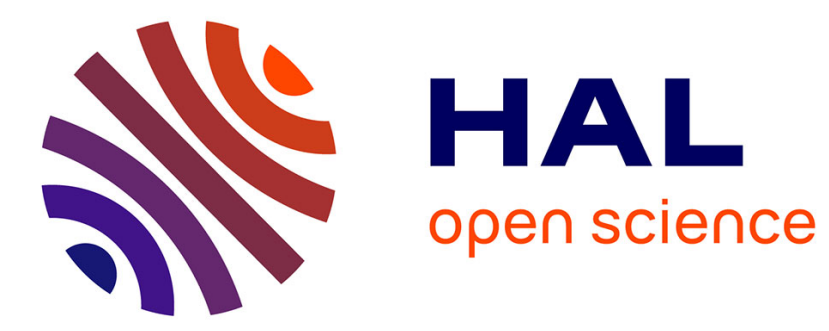

\title{
SURFACE PHONONS IN SILICON AND SURFACE RECONSTRUCTION
}

\author{
W. Goldammer, Wolfgang Ludwig
}

\section{To cite this version:}

W. Goldammer, Wolfgang Ludwig. SURFACE PHONONS IN SILICON AND SURFACE RECONSTRUCTION. Journal de Physique Colloques, 1984, 45 (C5), pp.C5-119-C5-124. 10.1051/jphyscol:1984517 . jpa-00224135

\section{HAL Id: jpa-00224135 https://hal.science/jpa-00224135}

Submitted on 1 Jan 1984

HAL is a multi-disciplinary open access archive for the deposit and dissemination of scientific research documents, whether they are published or not. The documents may come from teaching and research institutions in France or abroad, or from public or private research centers.
L'archive ouverte pluridisciplinaire HAL, est destinée au dépôt et à la diffusion de documents scientifiques de niveau recherche, publiés ou non, émanant des établissements d'enseignement et de recherche français ou étrangers, des laboratoires publics ou privés. 


\title{
SURFACE PHONONS IN SILICON AND SURFACE RECONSTRUCTION
}

\author{
W. Goldammer and W. Ludwig \\ Institute for Theoretical Physics II, University of Muenster, \\ Domagkstrasse 75, D-4400 Muenster, F.R.G.
}

\begin{abstract}
Résumé - Nous avons calculé les modes de surface dans le silicium pour une surface (111). Sauf dans la limite des grandes longueurs d'ondes, les modes dépendent beaucoup des constantes de couplage dans les deux premières couches près de la surface. Nous avons apporté une attention particulière à la recherche des modes mous de surface. Nous trouvons un mode qui devient mou pour un vecteur d'onde $\vec{k}$ très proche de celui qui conduit à une reconstruction $7 \times 7$.
\end{abstract}

Abstract - We have calculated the surface modes in Silicon at a (111)-surface. Apart from the long range limit, the modes depend strongly on the force constants in the first two layers near the surface. Special emphasis has been put on an investigation of soft surface modes. We find one mode which becomes soft very near the $\vec{k}$-point which leads to a $7 \times 7$-superstructure.

1. INTRODUCTION - Phonon surface states in Si have been calculated within various different models already 10 years ago/1-3/. All the models have in common that the lattice parameters (effective charges, force constants) near the surface have been changed in an appropriate way. To our knowledge, nothing is known about the true values of the force constants near a Si-surface. So all the calculations use effective parameters which include harmonic effects as well as temperature effects (anharmonic, quasiharmonic changes). The general features of the surface modes are always the same: surface modes already occur at a free surface with unchanged parameters; the more the parameters are changed (lowered) the more surface modes occur the polarization character being different. The long wave acoustic surface modes, of course, agree with the Rayleigh- or generalized Rayleigh-waves. The different models deviate only in quantitative aspects. If there are larger changes in the parameters, caused e.g. by temperature or just by introducing the surface, one mode becomes soft. Trullinger /1/ calculates the surface modes introducing effective charges in the surface and finds a soft mode which leads to a reconstruction corresponding to a $2 \times 1-$ or $7 \times 7$-superstructure depending on the details. Zimmermann and others $/ 2$, $3 /$ use different force constant models (rigid ion, shell, bond charge) and find a soft mode at the $2 \times 1-$ superstructure point. For computational reasons their calculations have been done at certain points in $\vec{k}-$ space only. Therefore we now have recalculated these models for all the $\vec{k}$-points in the Brillouin-zone. We also find the general features again; only quantitative details depend on the special model.

2.METHOD - Surface modes propagate with a 2 -dimensional $\vec{k}$-vector $\left\{k \bar{j}, k_{2}\right\}$. Introducing a 2 -dimensional surface adapted Brillouin-zone we have to rewrite the phonon-dispersion curves using

$$
\overrightarrow{\mathrm{q}}=\overrightarrow{\mathrm{k}}+r \overrightarrow{\mathrm{g}}_{3} \quad(\bmod \overrightarrow{\mathrm{G}}) \quad
$$

$\vec{q}$ is the bulk vector, $\vec{g}_{3}$ a vector perpendicular to the surface, chosen so that with $-1 / 2<\gamma \leqslant 3+1 / 2$ all the threedimensional $\vec{q}-v e c t o r s$ are obtained. Collecting all. the ideal lattice frequencies belonging to different $r$, but $\vec{k}$ fixed, we obtain frequency bands for the surface vec- 
tors $\vec{k}$. The surface modes, localized at the surface, have frequencies lying outside these bands (in general).

For every fixed $\vec{k}$ the lattice can be looked upon as a linear chain perpendicular to the surface. Then the force constants also have to be transformed (Fourier) in such a way to describe a $\vec{k}$-dependent effective linear chain. The surface is introduced by just cutting the force constants between two neighbouring layers which then corresponds to a linear chain with a free end (ideal surface). In addition the force constants are changed, too.

The calculation of the surface modes is performed by Green functions method. Consequently the Green function is also that of a $\vec{k}-d e p e n d e n t$ Iinear chain, being thus a function $G(\vec{k} ; \omega)$. Once a model for the ideal lattice modes is given, $G$ can be calculated numerically up to sufficient accuracy. The perturbation consists of the force constants cutted by introducing a free surface and eventually of the changes of the forces near the surface. This perturbation has to be transformed with respect to $\vec{k}$, giving a $\vec{k}$-dependent perturbation $J(\vec{k})$. Contrary to the theory of point-defects, J does not depend on w because there is no change in the masses here.

The solutions for the localized modes follow from

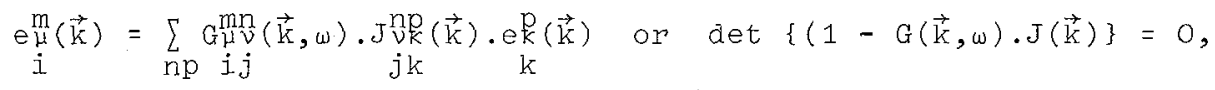

which gives the surface dispersion $\omega(\vec{k})$. m, $\mu, i$ denote the lattice geometry perpendicular to the surface (see above). The dimension of the determinant in (2) is that of the perturbation. In case of just cutting the force constants this dimension is low $(18 \times 18$ in our case, getting the surface modes twice because of our method).

Besides the proper surface modes calculated from (2) there may be resonance or quasi surface modes consisting of a solution of the homogeneous equation together with a special one of the inhomogeneous equation. These are scattering states, which may have a larger amplitude for the localized part than for the plane wave part. It is not easy to distinguish experimentally these resonance states from the strongly localized ones.

3.MODEL - The model we choose representatively (see above) assumes force constants between neighbouring atoms: centrai force constants up to fourth neighbours, non-central force constants between first and second neighbours.

$1^{\text {st }}$ neighbour:

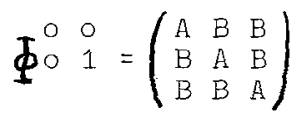
$2^{\text {nd }}$ neighbour:

$$
\begin{aligned}
& A=-1 / 3-8 \sigma / 3+8 \sqrt{2} \varphi / 3 \quad ; \quad a=-g / 2-\sigma / 6-\sqrt{2} \varphi / 3 \\
& B=-f / 3+4 \sigma / 3+2 \sqrt{2} \varphi / 3 ; \quad b=+2 \sigma / 3-2 \sqrt{2} \varphi / 3 \\
& 3^{\text {rd }} \text { neighbour : } \\
& \Phi_{0}^{0}{ }^{101}=-\frac{h}{11}\left(\begin{array}{rrr}
9 & 3 & -3 \\
3 & 1 & -1 \\
-3 & -1 & 1
\end{array}\right) ; \quad \Phi_{0}^{0} 0^{111}=-2\left(\begin{array}{lll}
1 & 0 & 0 \\
0 & 0 & 0 \\
0 & 0 & 0
\end{array}\right)
\end{aligned}
$$

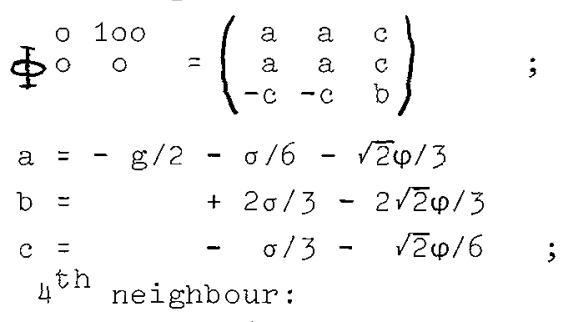

$\mathrm{f}, \mathrm{g}, \mathrm{h}, \mathrm{l}$ are central force constants, $\sigma, \varphi$ non-central (angle bending) constants. These constants are fitted to represent the ideal lattice modes appropriately, where special emphasis has been put to an overall agreement.

The surface has been described as an ideal one without changing any 
force constant near the surface (model A). Moreover further models have been introduced with surface force constants changed from which we discuss only a few (model B-E). It turned out that the frequencies of the surface modes are most strongly influenced by changes of the constants g", g"' which essentially describe the forces parallel to the surface. So we present those models having the largest changes in the constant $g$. In detail the force constants are given in the following table:
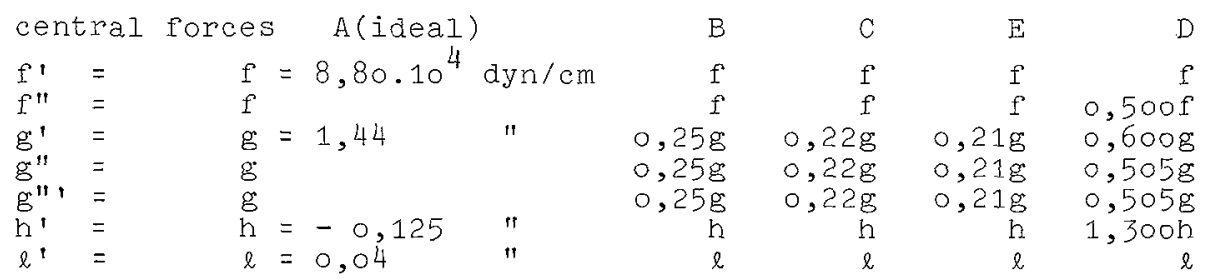

non-central forces

$\varphi^{\prime}=$
$\varphi^{\prime \prime}=$
$\sigma^{\prime}=$
$\sigma^{\prime \prime}=$
$\sigma^{\prime \prime}=$

$\varphi=-0,795 \quad "$
$\varphi=-0,02 \quad$ "
$\sigma=2$
$\sigma$

$\begin{array}{ll}\varphi & \varphi \\ \varphi & \varphi \\ \sigma & \sigma \\ \sigma & \sigma \\ \sigma & \sigma\end{array}$

$\begin{array}{ll}\varphi & 0,500 \varphi \\ \varphi & 1,500 \varphi \\ \sigma & 1,5000 \\ \sigma & 1,5000 \\ \sigma & 1,5000\end{array}$

The models $B, C, E$ specially show that small changes in the force constants change the frequencies strongly. Model D has very different force constants. But the surface modes calculated are not so different from those of the other models.

4. IDEAL SURFACE - In the long wave limit (acoustic) there is only one surface mode corresponding to the wellknown Rayleigh wave. Near the $\overline{\mathrm{K}}$-point there occurs a second surface mode, whereas others occur in the band gaps at higher frequencies; this is in agreement with former calculations /1-3/ though smaller deviations are present. In the following we are mainly interested in the acoustic surface modes. For this reason we have investigated the displacements connected with the different modes; in the long wave limit the polarization character is rather complicated due to the anisotropy of the surface. Whereas in the $\bar{T}$-direction we have a true Rayleigh-wave with a longitudinal and transverse ( $\perp$ surface) component, there is a generalized Rayleigh-wave with three components of polarization in the $\bar{\Sigma}$-direction. The amplitudes of the displacements decrease exponentially as it must be. But at the zone boundary the decay constant is comparatively large; at the $\bar{M}$-point, e.g. the amplitude has decreased in the $14^{t h}$ layer to only $10 \%$. This shows that one has to be careful when using layer-methods in the calculation of surface modes. 


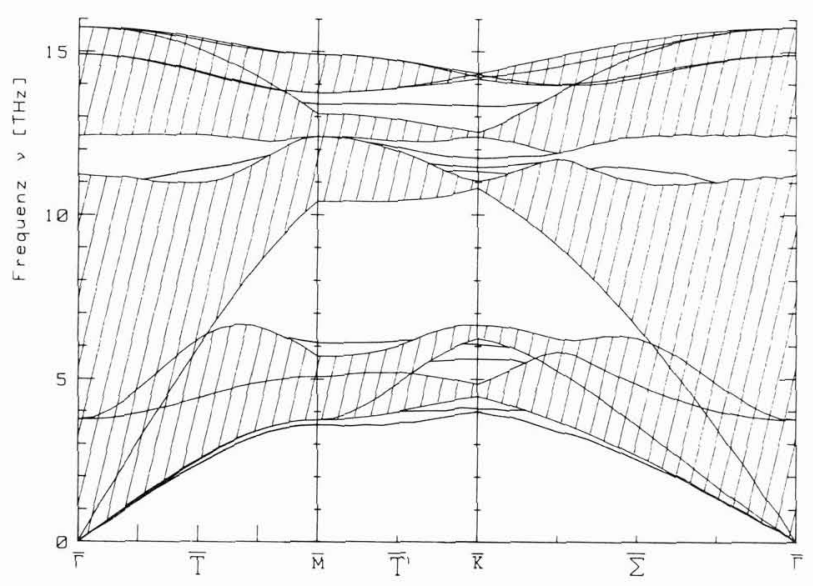

Fig. 1. Surface modes at a (111)-surface in Silicon with unchanged force constants in the surface, model A.

5.FORCE CONSTANTS CHANGED With weakening force constants the frequencies decrease, of course, and new surface modes occur. At higher frequencies surface modes may also vanish in the deeper Iying bands. In the models chosen we have three acoustic surface modes, but only one in the long range limit (Rayleigh limit). The others leave the bands at higher frequencies. The displacement vectors qualitatively show a similar behaviour as at anideal surface but with somewhat different polarizations and exponential decrease. The larger the changes in force constants, the stronger is the exponential decrease. In
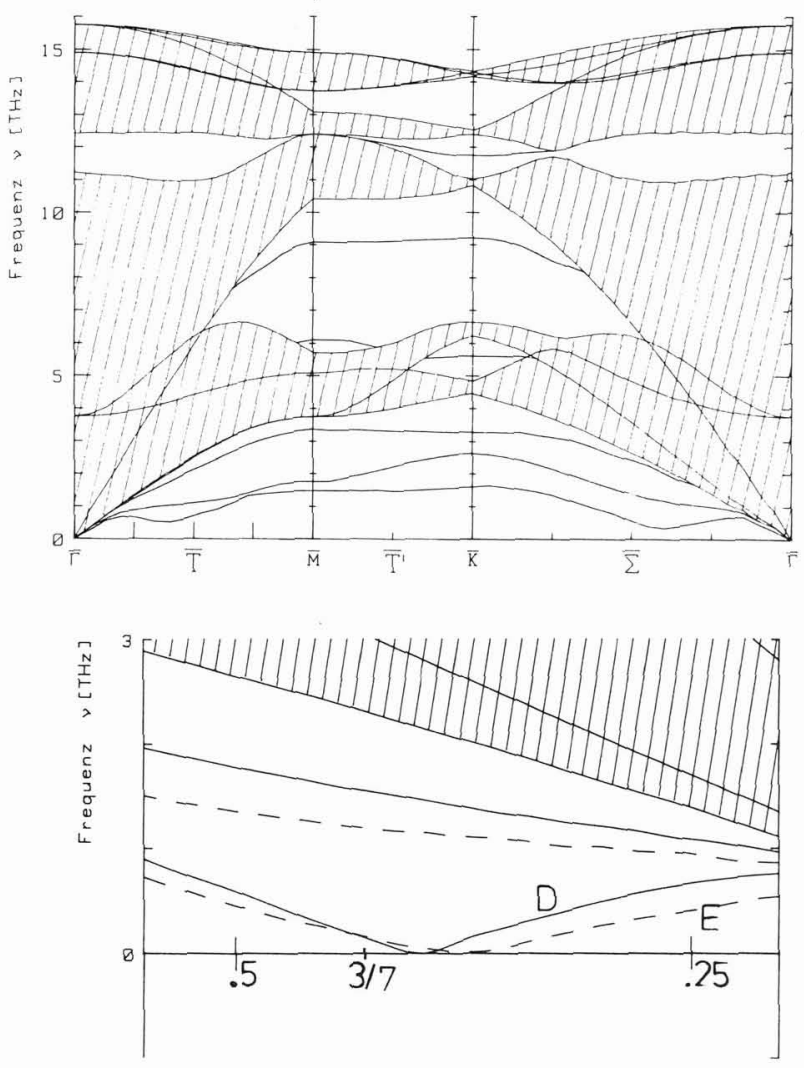

Fig. 2. Surface modes at a (111)-surface in Silicon with surface force constants changed, model $\mathrm{C}$

model $D, e . g$. for the $M-$ point a $10 \%$ decrease is already achieved in the $6^{\text {th }}$ layer. All the dispersion curves calculated show a more or less pronounced minimum in the $\tilde{\Sigma}$-direction, sometimes in the $\overline{\mathrm{T}}$-direction too, depending on the changes of force constants. With a certain amount of force constant change, the lowest mode frequency goes

Fig. 3. Surface modes in the neighbourhood of the $3 / 7$-point in the $\bar{\varepsilon}$-direction, model $\mathrm{D}$ and $\mathrm{E}$.

to zero (becomes soft) at a point in $\bar{\Sigma}$-direction, which is very near to $3 / 7$ of the $\bar{K}$-point. The point $3 / 7$ corresponds to $7 \times 7-r e-$ construction of the surface (see figs. 3,4). This soft mode has not been found in a previous calculation $12 /$ performed at

symmetry points only. In that calculation however a softening at the
M-point was found. This would now occur only when the force constants are changed even more and is not of interest here. 


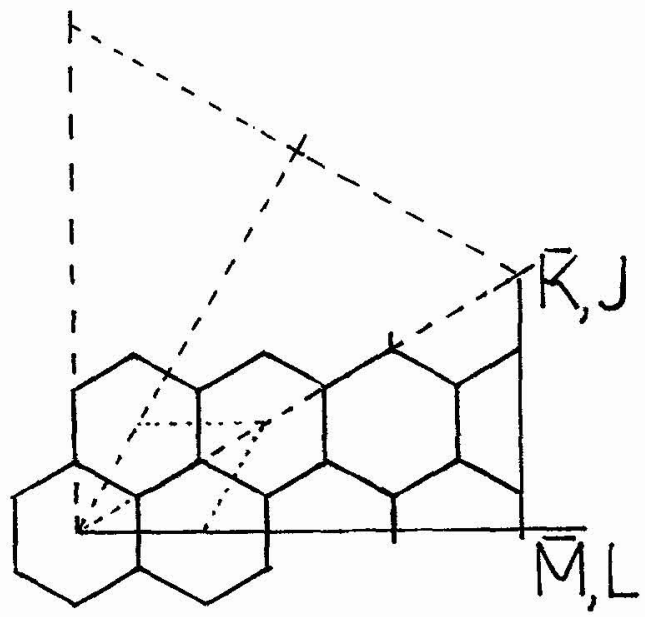

Fig. 4. Part of Brillouin-zone for the (111)-surface and reconstruction zones for the $7 \times 7-$ superstructure.

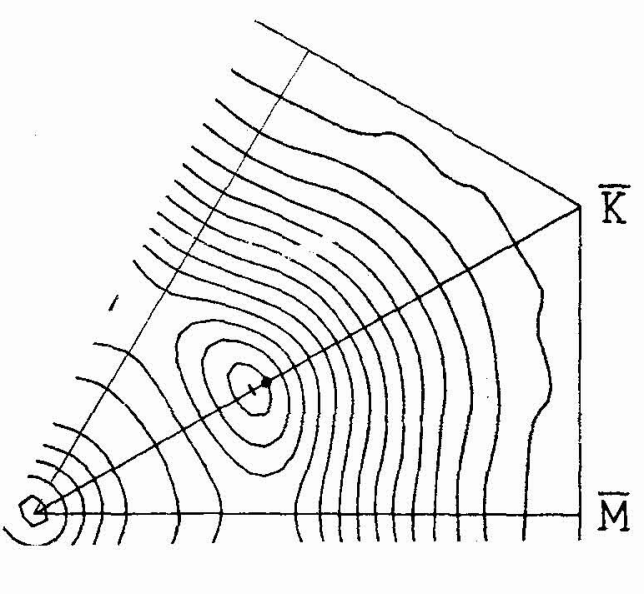

Fig. 5. Iso-frequency lines for the lowest acoustic surface modes, model $D$. Lines indicate a distance of $\Delta v=0.16 \mathrm{THz}$.

In all the calculations done the soft mode occurs exactly on the $\bar{\Sigma}-d i-$ rection which can be seen better from fig. 5 which shows iso-frequency lines over the surface Brillouin-zone. This is due to symmetry. But in no case the modes become soft exactly at the $7 \times 7-$ superstructure point, instead always slightly below this point, the deviation being about 3\% - 5,5\%. In Truliinger's /1/ model the mode becomes soft about 7\% above the $7 \times 7-p o i n t$. But there is a first softening at the $\vec{M}-p o i n t$, contrary to our calculations. A further hint for this softening is an electronic density calculation of Muramatsu and Hanke /4/. They find a strong increase of the response for $\vec{k}-v e c t o r s$ corresponding to a

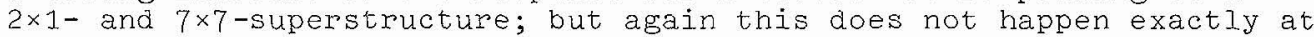
the $7 \times 7$-point.

The displacements behave very similar to those of other surface modes; especially the displacement vectors for the soft-mode-point and the exact $7 \times 7-p o i n t$ are nearly the same. Taking these displacements as the static ones which produce the new structure we obtain a lattice cell which is 49-times as large as the originaliy one. Since the $7 \times 7-p o i n t$ or the corresponding soft mode point has a 6 -fold degeneracy different displacement patterns are compatible with the soft mode concept. In fig. 6 we show the displacement pattern resulting from the most symmetric choice of the degenerate vectors, This pattern agrees very well

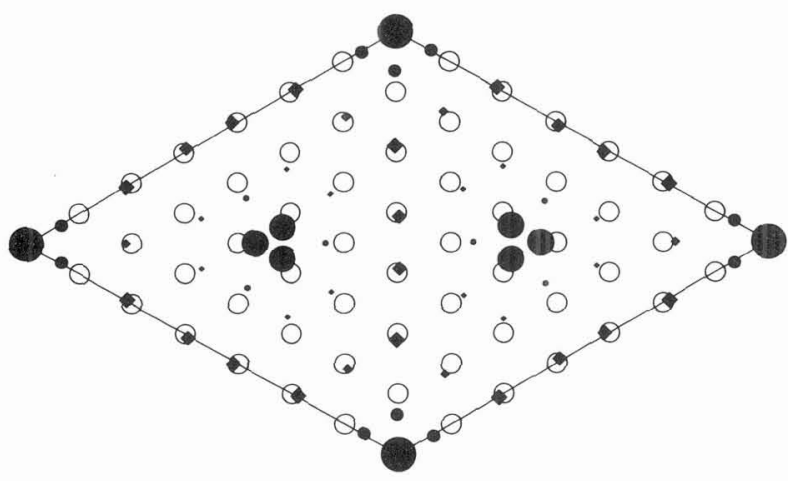
with one of those given by Chadi /7/.

Fig.6. Possible $7 \times 7$-surface reconstruction according to model D. Circles are atoms below the ideal surface, squares above (or vice versa). The magnitude of circles or squares, resp indicates the magnitude of the displacement.

Besides these there are other models; nearly a].I. these models have in common, that the displace- 
ments occur perpendicular to the surface, which seems to be proved by experimental results. Contrarily, our model allows for small displacement components paraljel to the surface, too. Whether these displacement components are real cannot be decided without further investigations. It should be mentioned, that the results of Binnig et al. /8/ cannot be reproduced with our model, because we start with a plane surface, whereas Binnig's results show a structure with different layers. Further, it might even be, that a 7×7-structure occurs with different patterns because of the degenercy of the soft points. Finally, if the deviation of the soft point from the $7 \times 7$-point is real, the structure given in fig. 6 is modulated in a large scale. This modulation is very weak, because the displacement vectors are practically the same and the modulation cell is about $200 \times 200$. Possibly the $7 \times 7$-structure is a geometrical effect; this assumption is supported by the experimental stability of this structure:

/1/ Truilinger,S.E. and S.L.Cunningham, 1973 Phys.Rev.Lett. 30, 913.

12/ Zimmermann,R., 1974 Applied Physics 3, 235.

13/ Hopster,H., 1974; Henzl,H., 1975 : Díplomarbeiten Münster.

14/ Muramatsu, A. and w. Hanke, 1982, Phys.Rev. B 27, 2609.

15/ Behnke,G., et a1., 1983, Phys. Verhandl. 4, 601.

/6/ Pollmann, J., 1983, Phys. Verhandl. $4,601$.

17/ Chadi,D.J., et aI., 1980, Phys.Rev.Lett. 44, 799;

1980, Surface Science $99,1$.

$18 /$ Binnig,G., et al., 1983, Phys.Rev.Lett. 50, 120. 